\title{
ESTUDIOS
}

\section{LA PRIMERA PESTE DE LOS ANTONINOS (165-170). UNA EPIDEMIA EN LA ROMA IMPERIAL}

\author{
Enrique Gozalbes Cravioto
}

Universidad de Castilla-La Mancha

\section{Inmaculada García García}

Universidad de Granada

\begin{abstract}
RESUMEN
El Imperio Romano tuvo una situación benigna desde el punto de vista sanitario en los primeros siglos de su existencia. Dicha situación sufrió una transformación a partir del año 164-165, con la irrupción de la denominada «Peste de los Antoninos». En el trabajo se estudia el desarrollo de esta epidemia, así como diversas interpretaciones acerca de la misma.
\end{abstract}

PALABRAS CLAVE: Imperio Romano, Epidemias, Crisis demográfica, Peste.

THE FIRST «ANTONINE PLAGUE» (165-170) A EPIDEMIC IN THE IMPERIAL ROME

\begin{abstract}
The Roman Empire had a benign sanitary situation in the first centuries of his existence. The situation suffered a transformation from year 164-165, with the irruption from the designated «The Antonine Plague». In the work is studied the development of this epidemic, as well as various interpretations about the same.
\end{abstract}

KEY WORDS: Roman Empire, Epidemics, Demographic crisis, Pest. 


\section{INTRODUCCIÓN}

Dentro de la serie de epidemias del mundo antiguo, nombradas como pestis en las fuentes latinas, ocupa una posición muy importante la que es conocida por la historiografía como «Peste de los Antoninos», o también como «Peste de Galeno». En cualquier caso, debemos aclarar que el termino latino pestis, o el de pestilentia, que equivalen al griego loímos, de acuerdo con Galeno corresponde a una enfermedad grave, con mucha frecuencia mortal, y que actuaba de forma simultanea sobre un gran número de personas. Este sentido clásico es el utilizado respecto a estas pandemias de la antigüedad, que no tienen que identificarse con la peste posterior, la peste bubónica ${ }^{1}$.

Hasta ese momento el estado sanitario de Roma había sido en general particularmente benigno, superando los fuertes brotes (la historiografía contemporánea considera que con toda probabilidad se trataba de malaria) de los siglos V al III a. C. Probablemente en ello había contribuido seriamente la introducción en la capital, y después en el Imperio, de la medicina científica griega $^{2}$. De hecho, las pestes acaecidas hasta ese momento en el Imperio Romano correspondían a episodios locales, de duración básicamente anual.

Esta es la característica principal de las pestes que estallaban en la propia Roma entre los siglos V al III a. C. Los datos que se reflejan acerca de las mismas señalan por un lado las creencias populares (y supersticiones), presentes a lo largo de la Historia en toda pandemia, pero también los intentos de una cierta observancia de las circunstancias y del desarrollo de la enferme$\mathrm{dad}^{3}$. No cabe duda de que estos últimos hechos también se debieron producir

1 Biraben, J. N. (1975), Les hommes et la Peste en France et dans les pays européens et méditerranéens, I, Paris, p. 22. En la definición de Isidoro de Sevilla sobre la peste se resume el saber clásico; ISIDORO, Etim. IV, 6, 18: Pestilentia est contagium, quod dum unum adprehenderit, celeriter ad plures transit. Gignitur enim ex corrupto aere, et in visceribus penetrando innititur. Hoc etsi plerumque per aerias potestates fiat, tamen sine arbitrio omnipotentis Dei omnino non fit (Ed. De J. OrOZ, Madrid, 1982). Más adelante el obispo hispalense habla de los bubones, puesto que refleja también ya la presencia del nuevo concepto de peste surgido con la epidemia de época de Justiniano; GozAlBES, E., GARCÍA, I. y RAMOS, M. C. (2005), «Enfermedad y cuidados en la obra de Isidoro de Sevilla», Index Enfermería, 51, pp. 70-73.

2 Thorwald, J. (1962), Histoire de la médicine dans l'Antiquité, Paris; EdelsteIn, L. (1967), Ancient Medicine, Baltimore; Flashar, H. (1971), Antike Medizin, Darmstadt; LaIN Entralgo, P. (1978), Historia de la Medicina, Barcelona; Lain EnTralgo, P. (dir.) (1972), Historia Universal de la Medicina, II, Barcelona; LóPEZ PIÑERO, J. M. (2002), La Medicina en la Historia, Madrid, entre otros muchos trabajos.

3 ANDRÉ, J. M. (1980) «La notion de Pestilentia à Rome: du tabou religieux à l'interpretation préscientifique», Latomus, 39, pp. 3-16. Para la peste en las civilizaciones del 
en la peste de los Antoninos, si bien los fuertes temores ante el contagio presentes en algunos médicos, y muy señaladamente en Galeno, condujeron a la pérdida de esa información. De ahí la conclusión de que la única solución ante la pandemia era la huida, pues la peste carecía de curación ${ }^{4}$.

\section{EL ORIGEN DE LA EPIDEMIA}

La benigna situación sanitaria de Roma se transformó de una forma bastante intensa a partir del año 165, ya en época del emperador Marco Aurelio. Un momento en el que el Imperio Romano se hallaba en su máxima etapa de desarrollo, y la población urbana y rural había alcanzado una calidad de vida relativamente elevada. Según Luciano de Samosata esta pestilencia tuvo su origen en Etiopía, desde donde llegó a Egipto, saltó a Asia, y en 165-166 llegó a Seleucia; el saqueo de Seleucia por los romanos ocasionaría que la peste se extendiera entre el ejército, y a través del mismo por todo el Imperio roma$\mathrm{no}^{5}$. ¿Se trata de un buen testimonio?

Podemos preguntarnos acerca de hasta qué punto Luciano aquí no atribuye a la Etiopía el origen, a partir de la creencia generalizada de que esa zona era la que daba origen a las pestilencias. El geógrafo Estrabon, siguiendo las indicaciones de Posidonio acerca del carácter insano de las zonas tórridas, consideraba que el lugar de origen de las pestes era Etiopía, al Sur de Egipto, debido al calor que daba lugar a la aparición de numerosos insectos ${ }^{6}$. Y Plinio consideraba Egipto como la madre de todas las afecciones de la piel que daban lugar a epidemias7. En este sentido, el texto de Luciano parece con bas-

Próximo Oriente y en Grecia, BYL, S. (1993), «La peste à l'aube de la civilisation occidentale», Les Études Classiques, 61, pp. 25-34.

4 JACKSOn, R. (1988), Doctors and Diseases in the Roman Empire, Tulsa; SigERIST, H. E. (1987), A History of Medicine. Primitive and Archaic Medicine, $2^{\mathrm{a}}$ ed., Oxford. Al respecto es muy significativo el texto de CELSO, De Medicina I, 10 (Ed. de la Loeb Classical Library, Londres, 1960). También muchos siglos más tarde el médico español Juan Sorapán de Rieros, que en 1616 publicó la «Medicina española en proverbios», comentaba la huída que consideraba imprescindible ante la peste, con una tripe letra 1: «huyr luengo, lexos y largo tiempo».

5 Luciano de Samosata, Quomodo Hist. conscr. 15 (Ed. De A. Lehugeur, Paris, 1876); Eutropio, Brev.Urb.Cond. VIII, 6 dice que la pestilencia la importaron de Siria las tropas de Lucio Vero, que volvían vencedoras de los partos (Ed. De J. HellegduARCH, $2^{\mathrm{a}}$ ed., Paris, 2002). Vid. RodríGueZ GonZÁLEZ, J. (2005), Diccionario de batallas de la Historia de Roma (753 a. C.- 476 d. C), Madrid, p. 575.

6 Estrabon XVII, 3, 10 (Ed. de Cambridge, 1996).

7 Plinio, NH. XXVI, 4 (ed. de A. Ernout y R. PAPIN), Paris, 1957. Sin duda se estaba refiriendo básicamente a la lepra. 
tante claridad una mera sátira acerca de vivencias poco concretas acerca de la peste, un simple reflejo literario mucho más que un texto con valor histórico ${ }^{8}$.

En cualquier caso, lo cierto es que la presión sufrida por el Imperio en las fronteras orientales en el año 162 había motivado el que Marco Aurelio mandara al corregente, Lucio Vero, con fuertes contingentes de tropas para expulsar a los partos, que habían irrumpido en Siria, y para recuperar Armenia y Mesopotamia. Pero la fatalidad hizo que la peste estuviera ya presente en el año 164 en Armenia, de acuerdo con el testimonio del historiador Dion Casio $^{9}$. Las tropas de Lucio Vero en combate en la zona comenzaron a sufrir los efectos de la terrible epidemia.

Según cuenta el biógrafo del emperador, circuló como versión de gran difusión que la epidemia había tenido su origen en el templo de Apolo en Babilonia, y que fue debida a que un soldado romano, por simple casualidad, rompió una arqueta de la que surgió el vaho de la pestilencia, el cual invadió a la nación de los partos y desde allí infectó al mundo entero: «la versión que circuló acerca de esta pestilencia es que la misma nació en Babilonia, en el templo de Apolo, en donde un soldado hundió por casualidad una arqueta, de la cual escapó un vaho de pestilencia que invadió al pueblo de los partos, y de alli se extendió por el mundo entero» ${ }^{10}$. Los rumores persiguieron al coregente, puesto que la peste se extendió después por todos los países por los que atravesaba en su camino de vuelta a Roma.

Ammiano Marcelino cuenta más o menos la misma historia milagrosa, con las supuestas emanaciones provocadas por una impiedad, y que ocasionarían una peste originada en Asia y extendida por todo el Imperio ${ }^{11}$. El tabú religioso continúa asociando el surgimiento de la peste a un fenómeno mágico. El propio hecho de creer que habían sido las emanaciones de un recipiente las causantes reflejan las creencias antiguas, acerca de la peste como corrupción del aire.

8 Hall, J. (1981), Lucian's Satire, Nueva York, pp. 312 y ss.

9 Dion Casio LXX, 3, 1 (Ed. de E. Cary, Cambridge, 1926); Gorlitz, W. (1962), Marc-Aurèle empereur et philosophe, Paris.

10 SHA., Vita Verus, VIII, 1: et nata fertur pestilentia in Babylonia, ubi de templo Apollinis ex arcula aurea, quam miles forte inciderat, spiritus pestilens evasit, atque inde Parthos orbemque complesse (ed. de D. MAGIE, Cambridge, 1967-1968). El templo se hallaba en la ciudad de Selevcia, que se encontraba junto a Tell Umar, ubicada a unos $30 \mathrm{kms}$. al Sureste de la actual Bagdad.

11 Ammiano Marcelino Hist. XXXI, 6, 24 (Ed. de J. C. RolFe), reimpr., Cambridge, 1972). 


\section{Desarrollo de la peste}

En el verano del año 165 la peste había cundido ya por Esmirna ${ }^{12}$. A finales de ese mismo año la pestilencia se había difundido por todo el Oriente romano $^{13}$. Los romanos diezmados por la enfermedad se vieron obligados a evacuar Mesopotamia14, y a concluir un tratado de paz con los partos. En el año 166 la peste estaba presente en Egipto, tal y como se refleja en un papiro; la extensión de la pestilencia por muchas comunidades egipcias durante cuatro o cinco años iba a tener efectos aniquiladores, que en algún caso llega al $40 \%$ de los habitantes de una localidad ${ }^{15}$.

Lucio Vero en su marcha de regreso hacia Roma, con sus soldados, fue viendo la transmisión de la epidemia; de acuerdo con lo escrito por su biógrafo, su mala fortuna hizo que llevara la peste a todas las provincias por las que pasaba hasta llevarla finalmente a la misma $\operatorname{Roma}^{16}$. No tiene nada de extraño, puesto que fueron sus soldados los que trasladaron la epidemia al Occidente, y los observadores de la época pudieron percatarse del hecho ${ }^{17}$. La recepción por parte de los dos emperadores de los títulos triunfales de «Pártico», «Arménico» $\mathrm{y}$ «Máximo» no evitó la constatación del papel de Vero en la transmisión de la peste.

En el mismo año 166 ya la peste se encontraba presente en Roma, pues es mencionada por Galeno, en su Methodus Medendi, quien dice que no permanecerá mucho tiempo probablemente a causa del desarrollo de la epidemia. Los efectos de la misma eran lo suficientemente devastadores, causantes de su huida ciertamente poco ejemplar, como para recordar la famosa peste de Atenas ${ }^{18}$. De hecho, el médico romano habla expresamente de una gran inflamación de los ojos, enrojecimiento muy fuerte del interior de la boca y de la lengua, sufrimiento por el paciente de una enorme sed, sensación de abrasamiento interior, enrojecimiento de la piel, tos violenta, erupciones y fistu-

\footnotetext{
12 Aelio Arístides Orat. XXXIII,6 y XLVIII, 38-39 (Ed. de M. Patillon, Paris, 2002).

13 DiON CASIO LXX, 3, 1.

14 DiOn CASIO LXXI,2,4.

15 BoAR, A. E. R. (1989), «Egypt and the Plague of Marcus Aurelius», Historia, 8, 1959, pp. 248-250. Vid. la discusión de los datos en SARTRE, M. (1994), El Oriente romano, Madrid, p. 458.

16 SHA., Vita Verus, VIII, 1: Fuit eius fati, ut in eas provincias, per quas redit, Romam usque luem secum deferre videretur.

17 Eutropio, Brev.Urb.Cond. VIII,6.

18 Galeno, Met. Med. XIX: in magna hac peste, cuius eadem facies fuit atque eius qua Thucydides memoria orassabatur (Ed. de Paris, 1530).
} 
las, seguidas de diarrea, agotamiento físico, etc. ${ }^{19}$, que son los síntomas descritos por Tucídides en el caso de la plaga ateniense.

Esta referencia de Galeno es la única que permite una cierta aproximación a los síntomas de la peste ${ }^{20}$. La comparación con las descripciones de Tucídides, más allá de la utilización de un modelo literario por parte de éste ${ }^{21}$. La descripción de Tucidides muestra datos interesantes sobre los síntomas en la peste de Atenas es relativamente amplia, señalando un inicio con cefaleas, enrojecimiento e inflamación de los ojos, ronquera y ataques de tos, vómitos y dolores estomacales, ematemas y ulceraciones cutáneas de grandes dimensiones; los que superaban esta situación sin fallecer veían como la afección pasaba a las extremidades, atacando los genitales y los dedos de manos y pies ${ }^{22}$. Más allá de los detalles, aparentemente ante la observación (superficial por su actitud ante la peste) de un médico como Galeno, en la peste de Roma se producían los mismos efectos.

En la biografía del emperador Marco Aurelio se refleja la llegada de la peste a Roma en el contexto de la guerra emprendida contra los marcomanos:

«Además se presentó una peste tan atroz que se tenían que sacar los cadáveres de la ciudad en vehículos y carretas. Fue entonces cuando los Antoninos sancionaron unas leyes estrictísimas sobre enterramientos y sepulcros, prohibiendo incluso que los particulares construyeran tumbas en sus villas, disposición que todavía hoy se cumple. La peste consumió a muchos millares y a muchos próceres, a los más ilustres de los cuales Antonio hizo erigir estatuas. Y tan grande fue su bondad que celebró funerales para las clases bajas corriendo las costas a cargo del Tesoro y perdonó a un cierto embaucador que arrestaron, y confesó en su presencia la tramoya que se había montado, con el fin de saquear la ciudad con algunos cómplices; en efecto, este falsario, encaramado en la higuera salvaje del Campo de Marte, se dedicaba a predicar que descendería fuego del cielo y se presentaría el fin del mundo, si él caía del árbol y se convertía en cigüeña. Y, claro está, en el momento oportuno cayó de la higuera salvaje y una cigüeña salió de su seno». ${ }^{23}$.

19 Galeno, Met. Med. XII.

20 Littman, R. J. y Littman, M. L. (1973), «Galen and the Antonine Plague», American Journal of Philology, 94, pp. 243-255. Vid. también recientemente, RiJKELS, D. F. (2003), «Pestis Antoniniana: enigma of diagnose», Geschiedenis der Geneeskunde, 9 (6), pp. 324-330.

21 AlsinA, J. (1987), «¿Un modelo literario de la descripción de la peste de Atenas?», Emerita, 55, 1987, pp. 1-13. Vid. también el estudio de RAMíREZ DE VERGER, A. (1985), «La Peste como motivo literario (a propósito de Coripo, Ioh. III, 338-379», Cuadernos de Filología Clásica, 19, 1985, pp. 145-156.

22 TuCídides II, 59, 2-8 (Ed. de Paris, 1962).

23 SHA,Vita Marcus Aurelius, XIII, 3-6: tanta autem pestilentia fuit, ut vehiculis cadavera sint exportata serracisque. Ttunc autem Antonini leges sepeliendi sepulchrorumque aspe- 
El grado de mortalidad en esos momentos era elevado, debido a la fortaleza de la epidemia, de forma que contra la piedad más usual la evacuación de cadáveres se producía de forma colectiva. Eran miles los fallecidos, lo que indica sin duda una incidencia especial en relación con otras pandemias de la antigüedad El propio testimonio de la prohibición de enterramientos en las villas, junto a la alusión al pago de los funerales de los pobres, indica una pandemia extendida entre todas las clases sociales. Y también se habla de la existencia del falsario apocalíptico.

También el biógrafo menciona un lectisternium que, como los más tradicionales anteriores al Principado, se debió efectuar en rogativas por la curación de la peste, y no tanto en petición de la victoria militar en Oriente (como señala esta fuente). Consistía dicha ceremonía en vestir las estatuas de algunos dioses y ofrecerles un banquete. El mismo biógrafo del emperador señalaba en términos elogiosos como en esta misma época Roma pudo emprender una nueva guerra victoriosa contra los marcomanos, en unos momentos en los que la peste ocasionaba miles de muertos tanto entre los soldados como entre los civiles ${ }^{24}$.

La gran peste iniciada en el 165 tenía dos características muy especiales, que la destacaban sobremanera respecto a los episodios mucho más limitados que habían acaecido en siglos anteriores. En primer lugar, que como señalaba Galeno era enormemente persistente, y ciertamente duraría varios años ${ }^{25}$. Pero el segundo elemento era además el de su enorme extensión, que afectó a buena parte del Imperio.

Por el Oriente, en invierno del 168 al 169 la peste estaba en Aquileia cuando allí se extiende entre las tropas concentradas en la plaza, cuestión que también documenta Galeno ${ }^{26}$. Por el Occidente la extensión de la epidemia fue parcial.

rrimas sanxerunt, quando quidem caverunt, ne quis ut vellet fabricaretur sepulchrum. quod hodieque servatur. Et multa quidem milia pestilentia consumpsit multosque ex proceribus, quorum amplissimis Antoninus statuas conlocavit. Tantaque clementia fuit, ut et sumptu publico vulgaria funera iuberet ecferri et vano cuidam, qui diripiendae urbis occasionem cum quibusdam consciis requirens de caprifici arbore in campo Martio contionabundus ignem de caelo lapsurum finemque mundi affore diceret, si ipse lapsus ex arbore in ciconiam verteretur, cum statuto tempore decidisset atque ex sinu ciconiam emisisset, perducto ad se atque confesso veniam daret.

24 SHA,Vita Marcus Aurelius, XVII,2: speciale ipse bellum Marcomannicum, sed quantum nulla umquam memoria fuit, cum virtute tum etiam felicitate transegit, et eo quidem tempore, quo pestilentia gravis multa milia et popularium et militum interemerat.

25 Galeno, V; X; XVIII.

26 GALENO XIX. 
De acuerdo con Ammiano Marcelino, esta peste procedente de Asia se habría extendido por todo el Imperio, alcanzando hasta el Rhin y la Galia ${ }^{27}$. Esta afirmación parece indicar que la peste no llegó a alcanzar en sus efectos ni las Hispanias ni el África romana ${ }^{28}$.

De hecho, se ha aducido en ocasiones que, de acuerdo con la biografía de Marco Aurelio, al encontrarse las Hispanias exhaustas por la peste se mandaron colonias de Italia a España ${ }^{29}$. ¿Hasta qué punto esta interpretación es correcta? En realidad el texto lo que dice es lo siguiente: «(tomó disposiciones a favor) de los hispanos, que estaban exhaustos por los reclutamientos de (colonos) itálicos, los cuáles iban en contra de los decretos promulgados por Trajano» ${ }^{30}$.

Este texto se encuentra absolutamente aislado del episodio de la guerra de Oriente, y también de la peste, y a nuestro juicio poco tiene que ver con ellas; lo que se documenta es que en ese momento las Hispanias se encontraban exhaustas debido a los continuos reclutamientos de los colonos (ciudadanos) itálicos de la misma, lo cual iba en contra de los decretos adoptados por Trajano. Así pues, los datos conocidos parecen indicar que la peste iniciada en el 165 no alcanzó las Hispanias.

Marco Aurelio ha pasado a la Historia como el último gran emperador de la etapa áurea, la de los Antoninos, de la Historia de Roma. El emperador que escribió su filosofía estoica no podía menos que tomar medidas frente a la epidemia en la propia capital. Estas medidas quedaron naturalmente en manos de la organización de la sanidad pública, y de la actuación de los médicos. Según el testimonio de su biógrafo, «como la peste todavía hacía estragos, restituyó celosamente el culto de los dioses, e instruyó para la milicia a los esclavos, como se había hecho en tiempo de la guerra púnica, a los que se llamó voluntarios como a los volones. También armó a los gladiadores a quienes llamó los complacientes, e hizo soldados de los bandoleros de Dalmacia y los Dardanelos $\rangle^{31}$. Las ceremonias religiosas constituían las respuestas más previsibles, pero las medidas para proporcionar más números al ejército, con la incorporación de esclavos y sobre todo de los bandoleros, iban a ser profundamente negativas en el futuro de las armas romanas.

27 Ammiano Marcelino Hist. XXXI, 6, 24.

28 LASSÈre, J. M. (1977), Vbique popvlvs. Peuplement et mouvements de population dans l'Afrique romaine de la chute de Carthage à la fin de la dynastie des Sévères (146 a. C.-235 p. C.), Paris, p. 552 destaca esta ausencia de testimonios a la afección de tan grave epidemia.

29 ZARAGOZA, J. R. (1971), Medicina y sociedad en la España romana, Barcelona, p. 178.

30 SHA,Vita Marcus Aurelius XI, 7: Hispanis exhausti(s) Italica allectiones contra... Tra(ia)nique proecepta verecunde consuluit.

31 SHA, Vita Marcus Aurelius XXI, 8. 
Lucio Vero falleció en el año 169 víctima de la peste (en Altinum). En algunos trabajos se apunta a que Marco Aurelio murió precisamente víctima de la peste. Este hecho se deduce de que en el momento de su fallecimiento, en el año 180, indicaba a los que lo acompañaban: "¿Por qué me llorais y no pensais más en la peste y en la muerte ante la que todos caeremos?» ${ }^{32}$. Entra en lo posible que, en efecto, la enfermedad del emperador viniera motivada por un rebrote de la peste en esas fechas, pero el hecho ni es seguro ni aceptado por una parte de los investigadores. En cualquier caso, esta infección estaría en relación no con la plaga del 165-170, sino con la del año 180.

En un trabajo reciente se ha puesto en relación la epidemia con la acuñación de las monedas en este tiempo, en concreto en lo que se refiere a las representaciones de carácter religioso. En efecto, en las monedas de Marco Aurelio se ha podido detectar que tanto en los ases, como dupondios, denarios y sestercios, indistintamente en los que llevan su nombre como el de Faustina, aparece representada la figura de la Salus, mientras en las cecas griegas orientales aparece representado con mucha frecuencia Esculapio, pero también en otras ocasiones Apolo, mismo fenómeno que se produce en las monedas con nombre de Lucio Vero. Estas referencias religiosas están con toda probabilidad en relación con la extensión de la peste por el Imperio ${ }^{33}$.

\section{CAMBIO DE CICLO: LA PESTE Y LOS CRISTIANOS}

Otras fuentes clásicas mencionan la peste de los Antoninos, si bien ofrecen muchos menos datos respecto a la misma. Según Orosio, la peste vino como consecuencia de un castigo divino debido a la persecución de los cristianos realizada por parte de Marco Aurelio, lo cual corresponde a un bien difundido topos de la polémica entre paganos y cristianos; la peste se extendió por muchas provincias, asolando especialmente Italia, afectando a los campos, a las villas, a las ciudades, de tal forma que algunos lugares quedaron desiertos ${ }^{34}$. Otro escritor cristiano, San Jerónimo, se limita a mencionar la peste de Antonino como lacra ${ }^{35}$.

32 SHA, Vita Marcus Aurelius XXVIII, 4: quid de me fletis et non magis de pestilentia et común morti cogitatis?

33 IdoATE, V. M. (2002), «Epidemias de peste y monedas en el Alto Imperio Romano», Gaceta Numismática, 147, 23-33.

34 Orosio VII, 15, 5 (Ed. de M. P. ARnaud-Lindet, Paris, 1991)

35 SAn Jerónimo, Chron., p.205 (Ed. Helm, Berlín, 1956). 
El cambio de ciclo de la situación de Roma se refleja bien en otros testimonios. La disminución de la población a consecuencia de las desgracias del periodo, como terremotos, inundaciones y sobre todo la gran peste Antonina ${ }^{36}$. Herodiano también refleja que hasta los tiempos de Marco Aurelio el Imperio no estaba acostumbrado a desgracias como los terremotos y las pestes ${ }^{37}$. A su juicio, el de un autor pagano, la época de Marco Aurelio marcaba la inflexión, el cambio de ciclo, de forma que de una etapa dorada para Roma y su Imperio, se pasaba a otra decadente y podríamos señalar que «enferma» ${ }^{38}$.

El testimonio de Tertuliano refleja una situación en la que los cristianos eran objeto de persecución, puesto que ante toda desgracia la población se volvía contra ellos: Si el Tiber desborda sus márgenes, si en Nilo no llega a los sembrados, si el cielo está inmóvil, si la tierra tiembla, si el hambre y la peste llegan, entonces gritais: cristianos al león» ${ }^{39}$. Por el contrario, Orosio de forma también apologética, como hemos visto, cambia los términos dialécticos, atribuyendo la peste al hecho de que los cristianos fueran perseguidos.

En cualquier caso, Marta Sordi, una de las mejores conocedoras de las persecuciones de los cristianos en el Imperio romano, ha considerado que a partir de los escasos datos conocidos, en la época de Marco Aurelio se detecta una etapa inicial de persecución en torno al $166 / 168$, debida sobre todo al prostagma o edicto sobre los sacrificios, promulgado debido a la peste; el recrudecimiento se produciría a partir del año 177, que se manifiesta en la gran persecución de Lyon $^{40}$. Aunque no existen referencias muy concretas a las persecuciones bajo Marco Aurelio, y la mayoría de los estudiosos niegan la existencia de una persecución general ${ }^{41}$, no cabe duda de que a medio plazo

36 EPITOME DE CAESARIBUS XVI,3 (Ed. de M. FESTY, Paris, 1999).

37 Herodiano I, 1, 4 (Ed. de Paris, 1990)

38 La bibliografía al respecto, con referencias al inicio y desarrollo de las epidemias es inmensa. Dentro de ella destacamos MAZzARINo, S. (1961), El fin del mundo antiguo, México, 1961; REMONDON, R. (1967), La crisis del Imperio Romano, de Marco Aurelio a Anastasio, Barcelona; FERNÁNDEZ UBIÑA, J. (1982), La crisis del siglo III y el fin del mundo antiguo, Madrid; BRavo CastañedA, G. (Coord.) (2001), La caída del Imperio Romano y la génesis de Europa, Madrid.

39 Tertuliano, Apol. 40, 2: si Tiberis ascendit in moenia, si Nilus non ascendit in arva, si coellum statit, si terra movit, si fames, si lues, statim: Cristianos ad leonem acclamatur (Ed. de J. P. WALTZING, Paris, 1971). Un siglo más tarde la propaganda pagana continuaba culpando a los cristianos de la expansión de la peste; CipRIANO, Ad Dem., 2. Vid. GonZÁlez SALINERO, R. (2005), Las persecuciones de los cristianos en el Imperio Romano, Madrid, p. 16.

40 SORDI, M. (1988), Los cristianos y el Imperio romano, Madrid, pp. 73-74.

41 Santos Yanguas, N. (1998), Cristianismo y sociedad pagana en el Imperio Romano durante el siglo II, Oviedo, pp. 86 y ss; GONZÁlEZ SALINERO, R. (2005), pp. 53 y ss. 
esta peste, y las sucesivas, iban a influir en la desesperación social, y en la aversión a los cristianos (como a partir de 1348 la Peste ocasionaría un fortísimo antijudaismo).

\section{CONSECUENCIAS E IDENTIFICACIÓN DE LA PESTE}

En algunos estudios de carácter más general se ha considerado que la Peste de los Antoninos fue un todo continuo que, iniciada en el 165 (o en el 164 en Seleucia) duró hasta el año 192, provocando la muerte del propio Marco Aurelio $^{42}$. Es natural que desde este planteamiento, a nuestro juicio en exceso superficial en algunos aspectos, la peste fuera realmente aniquiladora, con unos efectos similares a la Peste Negra del siglo XIV. No obstante, los datos conocidos apuntan en otra dirección diferente. El episodio surgido en el 165 aparentemente se agota en torno al 169-170, probablemente tiene un rebrote (o bien otra enfermedad diferente) en torno al 178-180, y tiene otro rebrote más fuerte (al menos en la capital) en el año 189.

El cambio de situación epidemiológica en Roma debe ponerse en relación con un factor apuntado para el siglo XIV, con la Peste Negra, la que Leroy-Ladurie ha llamado "unificación microbiana» ${ }^{43}$. En este caso que ahora nos interesa, el Imperio al haber puesto en comunicación todo el mundo en el entorno mediterráneo, desde Mesopotamia a Britania, desde la Selva Negra al Sahara, había provocado una especie de «globalización» de los bacilos y las bacterias.

En cualquier caso, debemos reflejar que esta unificación se había producido con mucha anterioridad, al menos desde la época del Principado de Augusto. La existencia de una epidemia «global», como elemento novedoso, tardó más de siglo y medio en producirse, cuando la Pax Romana hacía mucho tiempo que había abierto los mares a la navegación, y las calzadas a la comunicación terrestre. Por otra parte, el papel en la transmisión de la misma de los comerciantes no aparece expreso, por el contrario, fueron los soldados los que se infectaron, y los que en su vuelta expandieron la enfermedad. Duncan Jones ha analizado los efectos y circunstancias de la pandemia, destacando que los datos podrían indicar una mayor incidencia de la misma en las ciudades, debido a un mayor contacto de los soldados con la población de las mismas ${ }^{44}$.

42 Por ejemplo, en G. C. KoHN (ed.), Enciclopedia of plague and pestilente, Nueva York, 1995, p. 6, s. v. «Antonine Plague» (Plague of Galen).

43 LE Roy LADURIE, E. (1973), «Un concept: l'unification microbienne du monde (XIV siècle-XVII siècles», Scheweiz Z.Geschichte, 23, pp. 627-696.

44 Duncan Jones, R. (1996), «The impact of the Antonine plague», Journal of Roman Archaeology, 9, pp. 108-136. 
Respecto a la interpretación de las características y el alcance definitivo de la peste, la historiografía ha distado mucho de llegar a las mismas conclusiones. En el trabajo más completo acerca de la pandemia, que tiene ya cerca de medio siglo, Gilliam rebajó muy considerablemente el alcance real de la misma, tanto en lo referido a la cantidad de los fallecidos, como a sus efectos en la población del Imperio ${ }^{45}$. A su juicio habría que escapar de las exageraciones tendentes a centrar en la plaga antonina una de las causas principales de la crisis del Imperio romano.

Por el contrario, McNeill ha defendido que el episodio de peste iniciado en el 165, así como otros que le sucedieron, tuvo una mayor importancia de la que en ocasiones se le ha concedido. Marcaba el inicio de un giro en la situación demográfica del Imperio romano. Los escasos datos conocidos apuntan a que se trataba de una enfermedad nueva para las poblaciones mediterráneas, por lo que el comportamiento de las mismas fue similar al acaecido en otras ocasiones cuando la pandemia actuaba sobre poblaciones no inmunizadas. La mortalidad provocada fue particularmente elevada, entre un tercio y cuarta parte de la població, en un epsiodio que inició el declive continuo de la población en el marco del mundo mediterráneo ${ }^{46}$.

En concreto, McNeill plantea una interpretación muy sugerente a partir de la posibilidad de que la peste del año 165 , y las que le sucedieron, constituyeran la irrupción del sarampión y la viruela en el mundo mediterráneo. En efecto, estas enfermedades no parece que existieran como afecciones infantiles en la antigüedad, no existían en Egipto (como se ha podido certificar en el estudio de las momias de épocas muy diversas), ni tampoco hablan de ellas los médicos griegos y romanos: «los datos literarios apuntan así a los siglos II y III de la era cristiana como la época más probable en que ambas enfermedades se establecieron entre las poblaciones mediterráneas.... ambas enfermedades, sumamente contagiosas, irrumpieron una tras otra en las poblaciones comparativamente masivas, pero antes no afectadas, del mundo mediterráneo ${ }^{47}$.

No cabe duda de que el Imperio Romano vivió, en especial a partir de finales del siglo II, una situación problemática desde el punto de vista demográfico, y que la población del mismo (en especial en Occidente) tendió a disminuir. El problema por un lado consiste en la consideración del carácter

\footnotetext{
45 Gilliam, G. F. (1961), «The Plague under Marcus Aurelius», American Journal of Philology, 82, pp. 225-259.

46 MCNeILL, W. H. (1984), Plagas y pueblos, Madrid, pp. 116-117.

47 MCNEILL, W. H. (1984), pp. 118-119.
} 
catastrófico de este proceso ${ }^{48}$, en especial en lo que se refiere a la incidencia de la peste de los Antoninos. Baste indicar ahora que cuando en el año 189 penetre en Roma una nueva pandemia, que en la ciudad ocasionará hasta 2.000 muertos en un día, se indicará que esta enfermedad era la más grave de la que hasta entonces se había tenido noticia ${ }^{49}$, indicación de que la peste del 165-170 había sido algo o mucho menos grave.

Desde algunos estudios del siglo XIX se ha apuntado la posibilidad de que la peste de los Antoninos correspondiera a la viruela ${ }^{50}$. Esta opinión ha sido discutida por algunos investigadores más próximos, al señalar que las fuentes sobre la pandemia no ofrecen ningún dato que permita identificarla ${ }^{51}$. No obstante, ya hemos visto la opinión de McNeill que interpreta este episodio como la primera irrupción de la viruela entre las poblaciones del mundo mediterráneo, con una explicación que mostraría el episodio (y los siguientes) a la luz de la afección varios siglos más tarde en el Nuevo Mundo. También Mirko D. Grmek ha considerado que las pestes de la época romana imperial, siglos II y III, con toda probabilidad correspondían a la viruela, una enfermedad que tan solo recibiría nombre latino en el siglo VI ${ }^{52}$. En el mismo sentido, sin mayores argumentaciones, ha apuntado Symon Byl ${ }^{53}$, y como una posibilidad no del todo demostrada por Corvisier y Suder, en el más reciente estudio general sobre la población en la antigüedad ${ }^{54}$. En fechas más recientes, un buen trabajo de Rijkels ha insistido en la interpretación de la peste de Antonino como la irrupción de la viruela, a partir sobre todo de los síntomas recogi-

48 BoAK, A. E. R. (1955), «Manpower shortage and the fall of the Roman empire in the West», Ann. Arbor (Londres), pp. 15-21, ideas seguidas sin mayor profundización por CHAUNU, P. (1998), Historia y población. Un futuro sin porvenir, Madrid, 1998, pp. 65-66. Una contestación al análisis de Boak en FINLEY, M. I. (1975), «La cuestión demográfica», en A. M. PRIETO (ed.), La transición del esclavismo al feudalismo, Madrid, pp. 147-158.

49 Dion CASIO LXXII, 14, 3-4.

50 Hirsch, A. (1883), Handbook of geographical and historical pathology, I, Londres, p. 126. La mayor parte de la historiografía, especialmente la médica, a partir de los síntomas, interpreta muy verosímil la identificación de la pandemia con la viruela; BARQUET, N. (1997), «Smallpox. The triumph over the most terrible of the Minister of Death», Annals of Internal Medicine, 125, pp. 635-642, y sobre todo con una mayor profundización con anterioridad LitTMAn, R. J. y LitTMan, M. L. (1973); Gilliam, G. F. (1961). También considera la viruela G. C. KoHN (ed.), p. 6, que sigue sobre todo las conclusiones de McNeill.

51 Especialmente SendraIL, M. (1983), Historia cultural de la enfermedad, Madrid.

52 GRMEK, M. D. (1993), Les maladies à l'aube de la civilisation occidentale, Paris.

53 BYL, S., (1993), p. 31.

54 Corvisier, J. N. y Suder, W. (2000), La population dans l'Antiquité classique, Paris, p. 104. 
dos por Galeno, y aceptando en lo fundamental las conclusiones de McNeill sobre la importancia y alcance del brote epidémico ${ }^{55}$.

Por su parte Pierre Salmon, que recoge de forma algo más detallada los testimonios acerca de la peste de los Antoninos, también considera que la virulencia de la misma fue mucho menor que la de los episodios pandémicos posteriores. Respecto a la etiología de la peste, destaca la imprecisión absoluta en las fuentes, para indicar que sobre la misma en los escritos se habían planteado múltiples posibilidades: viruela, tifus exantemático, gripe, disentería, varicela, cólera, peste pulmonar, peste bubónica; a su juicio los datos recogidos imposibilitan la obtención de mayores conclusiones, aunque insiste en que los efectos sobre la población debieron ser relativamente escasos ${ }^{56}$.

En cualquier caso, la discusión acerca de los efectos y consecuencias de la plaga de los Antoninos en su conjunto continua vigente hasta nuestros días, motivando una producción bibliográfica bastante interesante ${ }^{57}$. Así el propio estudio ya citado de Duncan Jones, que menciona unos efectos y alcances muy diferentes según las zonas ${ }^{58}$, la discusión de Ehmig (derivada del trabajo anterior) acerca de las consecuencias de la peste ${ }^{59}$, el estudio de Bagnall que se plantea desde el análisis de la posible incidencia en la población y en la demografía ${ }^{60}$, otro trabajo de este mismo autor acerca de la afección en Egipto, a partir de los datos de un papiro ${ }^{61}$. Finalmente, en fechas todavía más recientes, el estudio de Fears, quien tras plantear la extensión geográfica de la pandemia, y su posible identificación con la viruela (o en su defecto con ántrax), admite su fuerte incidencia en la población, y en la decadencia del Imperio romano ${ }^{62}$. Aspectos en los cuales también ha insistido Rijkels ${ }^{63}$.

55 RiJkels, D. F. (2003), pp. 326-328.

56 Salmon, P. (1974), Population et dépopulation dans l'Empire romain, Bruselas, pp. 134-139.

57 En especial en relación con la interpretación del influjo de catástrofes en la Historia. Vid. como ejemplo, WeITZ, D. (1972), Famine and plagues as factors in the collapse of the Roman Empire in the third century, Nueva York.

58 DunCAN JONES, R. (1996), pp. 108-136.

59 Ehmig, V. (1998), «Die Auswirkungen der Pesten Antoninischer Zeit», Zeitschrift für Papyrologie und Epigraphik, 122, pp. 206-207.

60 BAgnall, R. S. (2002), «The effects of Plague: model and evidence», Journal of Roman Archaeology, 15, pp. 114-120.

61 Bagnall, R. S. (2000), «P. Oxy. 4527 and the Antonine Plague in Egipt: death or flight», Journal of Roman Archaeology, 13, pp. 288-292.

62 FEARS, J. R. (2004), «The plague under Marcus Aurelius and the decline and fall of the Roman Empire», Infect Dis Clin. North Am., 18, pp. 65-77.

63 RiJKels, D. F. (2003), pp. 329-330. 


\section{CONCLUSIONES}

En suma, los datos recogidos y analizados, así como la bibliografía disponible, solo permiten una aproximación parcial al conocimiento del episodio que estudiamos. Más allá de las consideraciones de carácter genérico, la afección del 165-170 tuvo un alcance muy limitado, en relación con otros episodios históricos posteriores. No obstante, su propia existencia e incidencia supuso una novedad para Roma, la entrada en un nuevo ciclo sanitario después de varios siglos en una situación bastante benevolente. La capital imperial había visto, como consecuencia de la globalización, la llegada de enfermedades cutáneas contagiosas, en especial la lepra, como novedad en época de Nerón. Ahora podía observar como, al igual que ocurría en el conjunto de Italia, cundía una peste procedente del exterior.

Naturalmente debemos huir, al igual que en el caso de la famosa Peste de Atenas, de la consideración como peste bubónica. La comparación de los sintomas por parte de Galeno puede responder a una evocación literaria, pero el hecho en palabras de un médico que era buen profesional, más allá de su huida por considerar rabiosamente mortal la pandemia, no deja de señalar la identificación de una similitud en los síntomas. Entre todas las enfermedades propuestas, sin duda la viruela es la que recoge, con diferencia, un mayor número de adeptos, siendo razonable concluir que probablemente fue en este momento, y en otras pandemias posteriores, cuando dicha afección tuvo fuerte incidencia inicial en las poblaciones mediterráneas, terminando por convertirse en endemia infantil.

Respecto a los efectos de las pestes, superadas las visiones (que rebrotan de vez en cuando) acerca de las catástrofes, a nuestro juicio la incidencia debe relativizarse. La crisis de Roma no se produjo por las epidemias, sino que existieron otras muchas razones que la explican con más verosimilitud. La misma crisis demográfica fue consecuencia al par que causa, debido a los problemas crecientes de subsistencia y alza de precios, junto a los cambios de mentalidad, y a una tradicional inestabilidad en una población de régimen demográfico antiguo. En este panorama, las pestes no vinieron sino a añadirse, aunque no fueran el factor principal, en un cambio de ciclo que significaría a la postre, mucho tiempo más tarde, el fin del mundo antiguo.

Fecha de recepción: 12 de junio de 2006

Fecha de aceptación: 10 de agosto de 2006 
